\title{
Cell-free synthesis of functional and endotoxin-free antibody Fab fragments by translocation into microsomes
}

\author{
Helmut Merk ${ }^{1}$, Christine Gless ${ }^{1}$, Barbara Maertens ${ }^{2}$, Michael Gerrits ${ }^{1}$, and Wolfgang Stiege ${ }^{1}$ \\ ${ }^{1}$ RiNA Netzwerk RNA-Technologien GmbH, Berlin, Germany, and ${ }^{2}$ Qiagen GmbH, Hilden, Germany
}

BioTechniques 53:153-160 (September 2012) doi 10.2144/0000113904

Keywords: cell-free protein synthesis; cell-free expression; antibody fragment; single-chain antibody; disulfide protein; Fab; antigen binding fragment

A eukaryotic cell-free system based on Spodoptera frugiperda cells was developed for the convenient synthesis of Fab antibody fragments and other disulfide bridge containing proteins. The system uses (i) a cell lysate that is mildly prepared under slightly reduced conditions, thus maintaining the activity of vesicles derived from the endoplasmic reticulum, (ii) signal peptide dependent translocation into these vesicles, and (iii) a redox potential based on reduced and oxidized glutathione. Monomeric heavy and light immunoglobulin chains are almost completely converted to highly active dimeric Fab joined by intermolecular disulfide bridges without supplementation of chaperones or protein disulfide isomerase. The applicability of the system is demonstrated by the synthesis of anti-lysozyme and anti-CD4 Fab antibody fragments yielding approximately $10 \mu \mathrm{g}$ Fab per milliliter reaction mixture. The lack of endotoxins in this system is a prerequisite that synthesized Fab can be applied directly using whole synthesis reactions in cell-based assays that are sensitive to this substance class. Moreover, the system is compatible with PCR-generated linear templates enabling automated generation of antibody fragments in a high-throughput manner, and facilitating its application for screening and validation purposes.

Monoclonal antibodies are used in a wide range of applications including as tools for research and for therapeutic purposes $(1,2)$. The range of possible applications is broadening rapidly with single-chain variable fragment $(\mathrm{scFv})$ and antigenbinding fragments (Fab), parts of complete antibodies that are small, expressible in Escherichia coli, alluding for screening as well as selection of high-affinity binding molecules (3). However, one of the most important limitations of antibody fragment production is the time needed for generating and analyzing binding efficiency and the effect on target molecules $(1,4,5)$. In recent years, a considerable acceleration-as well as a more economical generation - of antibodies was achieved through expression in E. coli cells (6). The synthesis of one Fab has been shown in insect cells in vivo (7). Cell-based methods of antibody production, however, require time and labor intensive cell cultivation. Moreover, for bacterial expression, additional work has to be performed to clone antibody-encoding genes into expression vectors (8).

In contrast, cell-free expression is now regarded as a promising alternative for overcoming limitations of cell-based methods. Reasons for that are most notably the clear improvement of productivity and economy, as well as the activity of the synthesized proteins in prokaryotic (9-11) and eukaryotic (12-16) cell-free systems in recent years. Modifications of some of these systems allowed the production of disulfide containing proteins including $\operatorname{scFv}(17,18)$. However, because of their better binding properties, Fabs are a more attractive than $\mathrm{scFv}$ (19).

Recently, cell-free expression of one Fab in an $E$. coli-based system has been shown $(20,21)$. However, disadvantages of $E$. coli-based cell-free systems include lower specific activity of produced $\mathrm{Fab}$ and the requirement for the removal of toxins present in the expression system before the synthesized Fab can be applied to cell-based assays. By now, cell-free $E$. coli systems in their simple format (batch) yield up to $1 \mathrm{mg}$ de novo synthesized protein per milliliter reaction. Nevertheless, the highest yield reported for cell-free synthesis of functional $\mathrm{Fab}$ is only $30 \mu \mathrm{g} / \mathrm{mL}$ (21). Despite the high productivity of $E$. coli-based cell-free systems, only a small percentage of the synthesized protein is usable. Moreover, in order to obtain accurate data, the active and inactive forms must be separated.

Cell-free E. coli-based systems exhibit a high-intrinsicactivity, which changes the redox potential during disulfide protein synthesis remarkably. This change reduces the activity of synthesized Fab. An inhibition of this change was achieved by chemical pre-treatment of the cell lysate prior to protein synthesis reaction $(22,23)$. However, this procedure is detrimental for the handling of the system since it requires an additional work step that is difficult to automate.

For the synthesis of noteworthy amounts of soluble and active Fab antibody fragments, E. coli-based systems are supplemented with protein disulfide isomerase (PDI) and chaperones like GroE and DnaK (17-18,20-21). The cell-free synthesis of disulfide proteins using an insect cellbased system has been shown. However, this system also requires supplementation of PDI and the synthesis of Fab has not been demonstrated so far (24).

Hence, for the synthesis of Fab antibody fragments, there is a need for an easy to handle and automatable cell-free expression system without the requirements for chemical pre-treatment and supplementation with chaperons and PDI, allowing the synthesis of Fab with high-specific activity. Ideally, such a system is free of endotoxins and compatible with rapidly generated linear templates produced by PCR (25), thus avoiding time consuming cloning of expression vectors. 


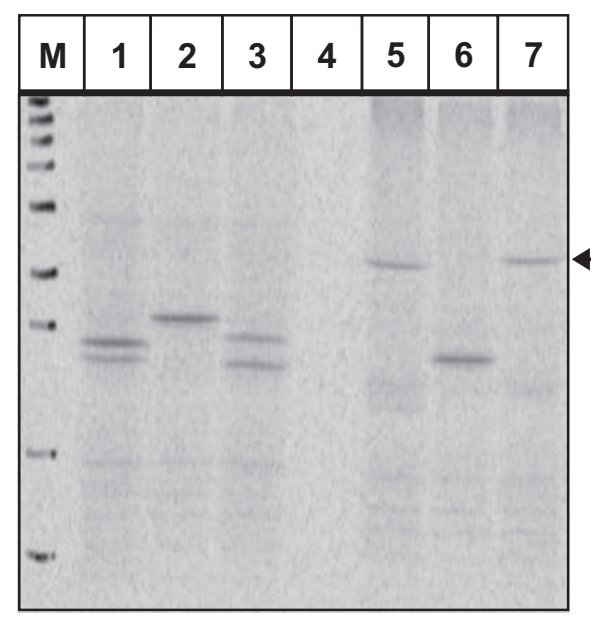

Figure 1. Autoradiography of SDS-PAGE analysis of Fab antibody fragment dimer formation. Reaction samples produced with the cell-free disulfide insect system were treated with sample buffer with (lanes 1-3) and without DTT (lanes 5-7). Lanes 1 and 5: coexpression of $\mathrm{L}$ - and $\mathrm{H}$-chains of anti-lysozyme Fab, lanes 2 and 6: expression of anti-lysozyme scFv as a control using pUTN3 as template (21) and in the presence of 0.5 $\mu \mathrm{M}$ PDI (Takara), lanes 3 and 7 coexpression of $\mathrm{L}$ - and $\mathrm{H}$-chains of anti-CD4 Fab. Lane $\mathrm{M}$ : molecular weight marker radioactively labeled after gel electrophoresis, from bottom 10, 15, 25, 35, 55, 72, 100, 130, $250 \mathrm{kDa}$. The arrow indicates the position of the Fab dimers.

\section{Materials and methods}

Generation of DNA templates

Primers were purchased from IBA (Göttingen, Germany). Molecular biology enzymes were from NEB (Frankfurt am Main, Germany). All Fab constructs, except controls, include the sequence for the honey bee melittin signal peptide. Genes encoding light and heavy chains ( $\mathrm{L}$ - and $\mathrm{H}$-chains) of HyHEL-10 anti-lysozyme Fab (26) were chemically synthesized by GeneArt (Regensburg, Germany) without their native signal peptide and with codon optimization for E. coli. Chemical synthesis also included an upstream sequence encoding honey bee melittin signal peptide and a sequence for the restriction enzyme recognition site $B s p \mathrm{HI}$ : 5'-GAGCTCATGAAATTCTTAGTC AACGTTGCCCTGGTTTTTATGG TGGTGTATATTAGCTATATTTATGCCGAT-3'. Following the translational stop codons, a sequence for $X h o \mathrm{I}$ was also included. The genes were subcloned into vector pIX5.0 (RiNA, Berlin, Germany), resulting in plasmids pIX5.0-Mel-LaLys and pIX 5.0-Mel-HaLys by using restriction enzymes $N c o I$ and $X h o I$ for the vector and $B s p \mathrm{HI}$ and $\mathrm{XhoI}$ for the genes.

pIX5.0-HaLys encoding the $\mathrm{H}$-chain of anti-lysozyme Fab without signal peptide sequence was constructed similar to pIX5.0Mel-HaLys, except that the melittin sequence described above was replaced by

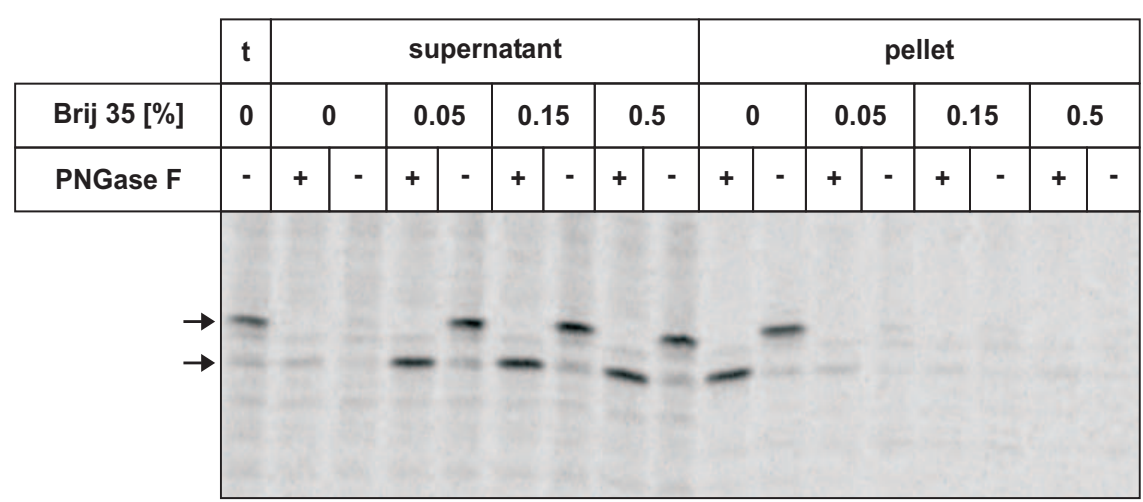

Figure 2. Autoradiography of SDS-PAGE analysis of release of synthesized protein from microsomal vesicles. For lysis of vesicles, reaction samples were treated with detergent and strong shaking for 5 min (Vibrax, IKA, 700 rpm), thereby avoiding air bubbles, followed by centrifugation for 10 min at $16,000 \times g$. t: total protein without centrifugation. Arrows indicate the position of glycosylated (upper) and deglycosylated Epo (lower).

5'-GAGCCCATGG and $N c o$ I was used for cloning instead of $B s p \mathrm{HI}$.

Mel-VLCL-SII encoding anti-CD4 (13B.8) Fab L-chain with a C-terminal Strep-tag and Mel-VHCH1 (H-chain) were produced as linear templates by Expression-PCR using the EasyXpress Linear Template Kit Plus (Qiagen, Hilden, Germany). The gene specific primers for H-chain 5'-GTATACATTTCTTACA TCTATGCGGACCAGGTTCAGCTGAAACAGTC-3' (forward), 5'-CTTG GTTAGTTAGTTATTAGCAAGAT TTGGGCTCAACTT-3' (reverse) and L-chain 5'-GTATACATTTCTTACAT CTATGCGGACGATATCCAGATGA CCCAGTC-3' (forward), 5'-GGATGA GACCAGGCAGAACACTCTCCGC GGTTGAAG-3' (reverse) and a StarGate vector containing the anti-CD4 (13B.8) sequence (IBA, Göttingen, Germany) as the template were used for the first PCR step according to the manufacturer's protocol. For construction of pIX5.0-MelEpo-N52+110Q encoding human erythropoietin (Epo) amino acid codons for asparagine 52 and 110 of pIX4.0-Epo (27) were changed to glutamine codons by site directed mutagenesis in order to inactivate two of the three $\mathrm{N}$-glycosylation sites of the protein. Using PCR, the sequence encoding the native signal peptide of Epo was replaced by a sequence for honey bee melittin signal peptide and restriction sites for $\mathrm{XbaI}$ and $B g l$ II were introduced using the forward primer 5'-GATAAATCATGAAATTCTTAGTCAACGTTGCCCTTGTTTTTATGGTCGTATACATTTCTTACA TCTATGCGGACCCACCACGCCTC ATCTGTG-3' and reverse primer 5'-TTA TTTAGATCTTTATCTGTCCCCTGT CCTG-3'. The PCR product was subcloned into pIX5.0 using restriction enzymes $\mathrm{NcoI}$ and $X h o I$ for the vector and $B s p \mathrm{HI}$ and $X h o \mathrm{I}$ for the gene.
Preparation of cell lysate and protein synthesis reaction Cell lysate of Spodoptera frugiperda was prepared as described (15), except that the elution buffer for chromatography of the supernatant of centrifugation did not contain the reducing agent DTT (dithiothreitol). The lysate was incubated with a final concentration of $6.9 \mathrm{U} / \mathrm{mL} \mathrm{S7} \mathrm{micro-}$ coccal nuclease and $1 \mathrm{mM} \mathrm{CaCl}$ (all from Roche, Mannheim, Germany) for $20 \mathrm{~min}$ at $20^{\circ} \mathrm{C}$. Incubation was stopped by supplementation with 5 mM EGTA (Sigma-Aldrich, Taufkirchen, Germany) and cooling on ice. Coupled transcription/translation reactions consisted of $35 \%(\mathrm{v} / \mathrm{v})$ nuclease treated cell lysate and $30 \mathrm{mM}$ HEPES-KOH (pH 7.6), $2.9 \mathrm{mM} \mathrm{Mg}(\mathrm{OAc}), 75 \mathrm{mM} \mathrm{KOAc}$ (all from Sigma-Aldrich), $0.25 \mathrm{mM}$ spermidine (Serva, Heidelberg, Germany), $20 \mathrm{mM}$ creatine phosphate, $1.75 \mathrm{mM}$ ATP, $0.3 \mathrm{mM}$ each of CTP, GTP and UTP (all from Roche), 0.33 mM pl-(guanosyl)p3-[5' (guanosyl)] triphosphate (Sigma-Aldrich), $2.5 \mathrm{mM}$ oxidized glutathione, $0.5 \mathrm{mM}$ reduced glutathione (Roche), $100 \mathrm{U} / \mathrm{mL}$ RNase inhibitor RNasin (Promega, Mannheim, Germany), $50 \mathrm{U} / \mathrm{mL}$ T7 RNA polymerase, $17.5 \mu \mathrm{g} / \mathrm{mL}$ baker's yeast tRNA (all from Roche), $0.1 \mathrm{mM}$ each of the 19 naturally occurring amino acids (Merck, Darmstadt, Germany) except leucine and 0.1 $\mu \mathrm{g} / \mathrm{mL}$ creatine kinase (Roche). For single expression $15 \mu \mathrm{g} / \mathrm{mL}$ plasmid DNA and for coexpression of light and heavy chains from different templates $7.5 \mu \mathrm{g} / \mathrm{mL}$ of each plasmid was used. The reactions were supplemented with $0.1 \mathrm{mML}-\left[\mathrm{U}-{ }^{14} \mathrm{C}\right]$ leucine (PerkinElmer, Rodgau, Germany) with a molar activity of $0.667 \mathrm{~Bq} / \mathrm{pmol}$. Reactions were incubated $4 \mathrm{~h}$ at $25^{\circ} \mathrm{C}$.

Quantification of the synthesized protein and gel electrophoresis

Protein quantification was done by trichloro acetic acid precipitation of radioactively labeled 
A

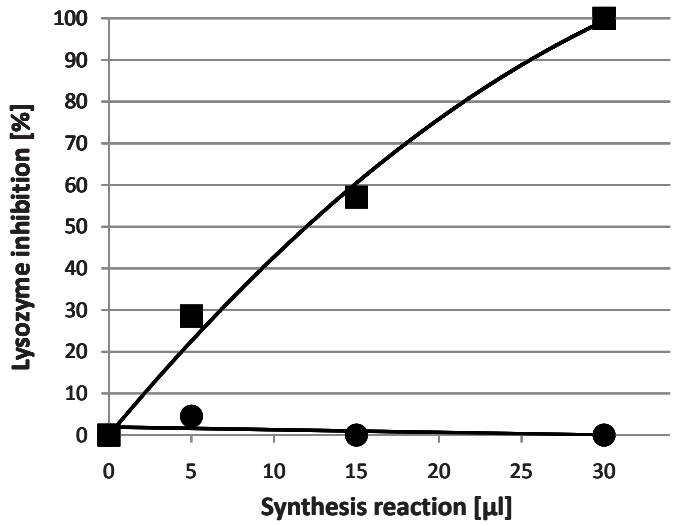

B

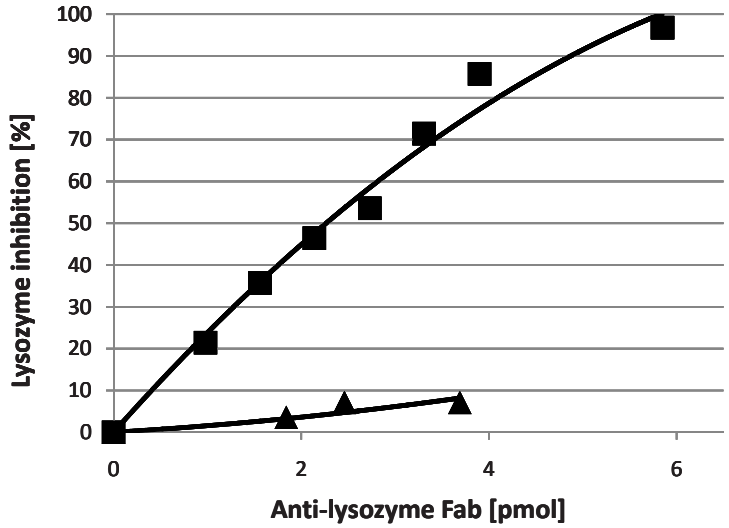

Figure 3. Activity measurements of anti-lysozyme Fab by inhibition of lysozyme. (A) Activity of anti-lysozyme Fab using the standard and the disulfide insect systems. Activity of Fab was measured as described in Materials and methods. Fab synthesis was performed using the disulfide insect system ( $\square$ ) and the standard system $(\bullet)$. In both cases, the synthesis was directed into microsomal vesicles. (B) Activity of anti-lysozyme Fab dependent on endogenous microsomes. Yield of synthesized Fab was determined for the disulfide insect system to $10 \mu \mathrm{g} / \mathrm{mL}(\mathbf{m})$ and for the microsome depleted disulfide insect system to $4 \mu \mathrm{g} / \mathrm{mL}$ ( $\boldsymbol{\Delta}$ ) measured by incorporation of a radioactively labeled amino acid. Microsome depletion was done by centrifugation of the cell lysate for 10 min at $16,000 \times g$ and $4^{\circ} \mathrm{C}$ prior to protein synthesis.

protein followed by scintillation counting as described (18). Autoradiography of SDS gels was performed as described (18) using a Typhoon imager (Amersham Pharmacia, Freiburg, Germany). A sample buffer without reducing agent was used for non-reducing electrophoresis and the samples were incubated $30 \mathrm{~min}$ at $37^{\circ} \mathrm{C}$ instead of boiling.

\section{Activity assay}

For calculation of the activity of anti-lysozyme Fab, 20 ng hen egg-white lysozyme (SigmaAldrich) with a specific activity of $56,400 \mathrm{U} /$ $\mathrm{mL}$ was incubated with different volumes of reaction samples after lysis of microsomes using detergent as described in Results and discussion. Specific inhibition of lysozyme dependent lysis of Micrococcus lysodeicticus cells (Sigma-Aldrich) was measured as described previously (18).

\section{Proteinase K protection assay}

After transcription/translation, six microliter reaction samples were incubated $10 \mathrm{~min}$ at $25^{\circ} \mathrm{C}$ with strong shaking in the absence or presence of $0.05 \%$ Bri35 (Thermo Scientific, Rockford, IL, USA) that is needed for lysis of microsomal vesicles. The samples were further incubated for $20 \mathrm{~min}$ at $25^{\circ} \mathrm{C}$ in the absence or presence of 0.1 or $0.5 \mathrm{mg} / \mathrm{mL}$ proteinase $\mathrm{K}$ (Roche) followed by addition of $5 \%$ trichloro acetic acid, incubation for $10 \mathrm{~min}$ at $56^{\circ} \mathrm{C}$ and $30 \mathrm{~min}$ precipitation on ice. After centrifugation at $16.000 \times g$ for $30 \mathrm{~min}$, pellets were washed with ice-cold acetone, dried, and dissolved in sample buffer for SDS-PAGE. Analysis was done by SDS-PAGE and autoradiography of the radioactively labeled proteins.

\section{Results and discussion}

The synthesis of Fab was performed by coexpression of immunoglobulin L- and $\mathrm{H}$-chains using the method and components previously described, henceforth called the disulfide insect system. For the expression of anti-lysozyme Fab, two plasmids (pIX5.0Mel-LaLys and pIX5.0-Mel-HaLys) encoding $\mathrm{L}$-and $\mathrm{H}$-chains were constructed via conventional cloning. Two linear templates, Mel-VLCL-SII (L-chain) and Mel-VHCH1 (H-chain), were produced for the synthesis of anti-CD4 Fab via expression PCR. In contrast to procedures described earlier, we provided the $\mathrm{L}$ - and $\mathrm{H}$-chains with a signal peptide in order to direct the synthesis into microsomal vesicles.
Gignalchem Specialists in Signaling Proteins Leader for Cell Signaling Products

\section{wvwv.signalchem.com}

T: 604.232.4600 1.866.9.KINASE F: 604.232.4601
To determine whether Fab antibody fragments are produced as dimers of $\mathrm{L}$ - and H-chains, anti-lysozyme Fab and anti-CD4 Fab were expressed using the disulfide insect system as described previously. After cell-free coexpression, reaction samples were separated by reducing and non-reducing $15 \%$ SDS-PAGE, the latter to maintain the integrity of the intermolecular disulfide bonds. The radiolabeled immunoglobulin chains were detected by autoradiography. Whereas the chains expressed in separate synthesis reactions do not form self dimers (data not shown), the co-expressed Land $\mathrm{H}$-chains are detected as dimers with increased apparent molecular weight in the non-reducing part of the gel (Figure 1). The conversion from monomeric to dimeric Fab chains is almost complete.

As the proteins that are synthesized in the disulfide insect system are expected to be translocated into microsomal vesicles by means of the melittin signal peptide, a direct interaction of these proteins with their target molecules is not possible without disruption of the vesicular lipid membrane. Therefore, we examined how a protein that has been translocated into vesicles can be made accessible to an application. Since $\mathrm{N}$-glycosylation is seen as a proof for previous translocation, we used the glycoprotein Erythropoietin (Epo) as a model to analyze the translocation into the vesicles and the subsequent release of synthesized proteins by treatment with detergent.

A radioactively labeled Epo mutant containing the melittin signal peptide and one $\mathrm{N}$-glycosylation site was expressed using the plasmid pIX2.0-Mel-Epo-N52+110Q as described in Materials and methods. Glycosylation was verified by enzymatic deglycosylation of reaction samples as described (27). After Epo synthesis, reactions were treated with different concentrations of detergent and subsequently centrifuged at $16.000 \times g$ 
for $10 \mathrm{~min}$. Under these conditions the vesicles including trapped Epo were pelleted, whereas after successful lysis the protein should remain in the supernatant. Indeed, at a concentration of $0.05 \%$ Brij 35 the vesicles are completely lyzed as indicated by the complete release of Epo and its recovery in the supernatant. Previous translocation into microsomal vesicles is indicated by the N-glycosylation (Figure 2).

Next the biological activity of Fab synthesized with the disulfide insect system was analyzed by the ability of anti-lysozyme Fab to inhibit the lytic activity of lysozyme. As a control, co-expression was performed in a commercially available standard system that also contains active microsomal vesicles but exhibits reducing conditions (EasyXpress Insect Kit II, Qiagen). As expected, activity of anti-lysozyme Fab can only be detected, if Fab is synthesized using the disulfide insect system containing oxidized and reduced glutathione, whereas there is no activity resulting from synthesis using the standard system (Figure 3A).

Since we provided the Fab chains with signal peptides for translocation into vesicles these results show that active Fab can be produced in cell-free systems using the combination of redox conditions and the natural translocation mechanism, and that translocation into microsomal vesicles might be useful for the synthesis of active Fab in such systems.

In order to examine the contribution of translocation to Fab activity, we removed the microsomes mostly from the disulfide insect system by centrifugation prior to protein synthesis. Overall synthesis yield was measured via incorporation of radioactively labeled amino acids as described in Materials and methods, and the specific activities of antilysozyme Fab synthesized in the presence and absence of microsomes were analyzed. For that purpose, the inhibiting effect of Fab on lysozyme activity was measured. Fab synthesized in the presence of microsomes shows activity, which is approximately one order of magnitude higher compared with Fab synthesized without microsomes (Figure 3B).

Next we examined whether one of the cell-free synthesized Fab chains is protected against protease digestion and whether such a protection is only possible if the chain has been synthesized with signal peptide. A protection only for signal peptide bearing Fab in the disulfide insect system would give evidence that it has been specifically translocated into microsomes where it is inaccessible for the protease.

The H-chain of anti-lysozyme Fab synthesized with signal peptide using the template pIX 5.0-Mel-HaLys and without signal peptide using pIX5.0-HaLys was subjected to the proteinase $\mathrm{K}$ protection assay. To force digestion, vesicles of control reaction samples were lysed by pre-treatment with detergent as

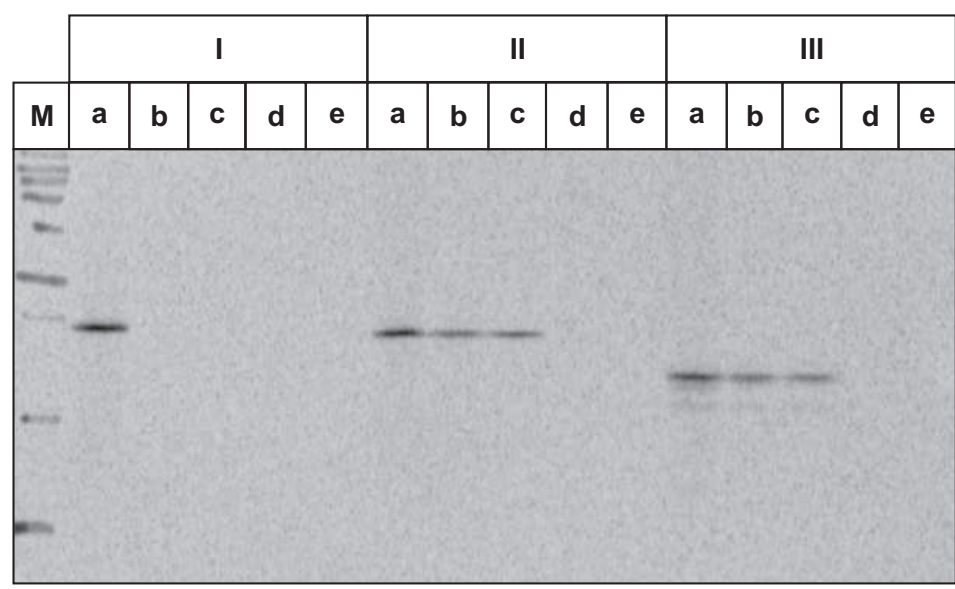

Figure 4. SDS-PAGE analysis of protease protection assay. Templates used for protein synthesis I: pIX5.0-HaLys (without sp), II: pIX5.0-Mel-HaLys (with sp), III: pIX2.0-Mel-Epo-N52+110Q (with sp). Lane a: reaction samples were treated with water (control), lanes $b$ and d: treatment with $0.1 \mathrm{mg} / \mathrm{mL}$ proteinase $\mathrm{K}$, lanes c and e: treatment with $0.5 \mathrm{mg} / \mathrm{mL}$ proteinase $\mathrm{K}$. Samples of lanes $\mathrm{d}$ and e were additionally treated with detergent for lysis of vesicles before addition of protease. Lane M: molecular weight marker as in Figure 1, sp: signal peptide.

described in Materials and methods.

Our results show clearly that the $\mathrm{H}$-chain of anti-lysozyme Fab is protected against protease digestion when synthesized with a signal peptide and completely digested if produced without a signal peptide. In addition, the protection of the signal peptide bearing $\mathrm{H}$-chain is disabled when the vesicles have been lysed by pretreatment with detergent. We conclude that the synthesized protein is specifically translocated into the microsomal vesicles (Figure 4).

Furthermore, to exclude that the microsomes have a nonspecific positive effect on Fab activity, we synthesized the Fab chains without signal peptides and measured Fab activity. As expected, anti-lysozyme Fab expressed without encoded signal peptide is not active in the disulfide insect system (data not shown). In summary, our results indicate that both redox conditions and translocation into microsomes are required for the synthesis of Fab with high activity.

In cell-free expression systems published to date, synthesis of Fab takes place in prokaryotic cell lysates in an environment corresponding to the cytosol of a living cell. Chaperones and PDI necessary for the synthesis of active Fab are supplemented to these systems. In the eukaryotic system described here, the antibody fragment encoding templates contain a sequence for a signal peptide. By means of the signal peptide, the antibody fragments are cotranslationally translocated by a natural mechanism into microsomal vesicles derived from the ER, which are highly active because of gentle preparation of the cell lysate. This kind of synthesis is more similar to the natural synthesis of antibodies in B cells than a cytosolic synthesis. Most likely the microsomal vesicles already contain all the components such as chaperones and disulfide isomerase required for the maturation of antibody fragments, so there is no need for a balanced supplementation of all these components.

In current systems, a certain redox potential can only be maintained for a limited period of time because of enzymatic processes generating a more reducing environment that is disadvantageous for disulfide bond formation. Hence, in order to inhibit these enzymes, such systems are pretreated chemically, for instance, with an excess of oxidized glutathione or iodoacetamide. In the system described here, the redox potential seems to be sufficient for the almost complete conversion of monomeric immunoglobulin chains to dimeric Fab. A possible reason for that seems to be the natural capability of the ER to maintain its redox potential. We assume that this potential is still present in the microsomal vesicles.

Present prokaryotic systems contain endotoxins. Thus, antibody fragments produced in such systems cannot be used directly for applications involving endotoxin sensitive eukaryotic cells, as cytotoxic effects interfere with the measurement of the specific impact of antibodies on cells. Using the eukaryotic endotoxin-free system presented here, a purification step after protein synthesis for removal of endotoxins can be avoided.

So far, mRNA or circular DNA are mostly used as templates for cell-free protein synthesis. With the system described here, linear template DNA produced by PCR can be applied even without prior purification. During the process of template generation, sequences encoding the signal peptide required for translocation and affinity tags are easily added to the open reading frames of the immunoglobulin chains. Together with the avoidance of chemical pretreatment 
of cell lysates and the lack of necessity for the removal of endotoxins, the potential to use linear templates without the need of time-consuming plasmid construction is an ideal basis for the automized production of antibody fragments and other disulfide proteins for screening purposes in a high throughput manner.

Although prokaryotic cell-free systems usually yield up to one milligram de novo synthesized protein per milliliter reaction, to date the production of only $30 \mu \mathrm{g}$ active Fab per milliliter has been reported (24). The consequence of a low percentage of active Fab for applications that may be negatively affected by the presence of inactive portions, is that the inactive portion has to be removed. Such additional purification steps complicate automation of antibody fragment production markedly. Although the yield of synthesized Fab is comparatively low, the specific activity of antibody fragments produced with the expression system described here is much higher compared with present prokaryotic systems since Fab is produced predominantly in active form. We conclude that purification of $\mathrm{Fab}$ from the disulfide insect system in order to remove inactive immunoglobulin chains is obsolete.

\section{Acknowledgments}

This study was supported by a grant from the Bundesministerium für Wirtschaft und Technologie (BMWi, No. ZIM EP091944) and partially by the Bundesministerium für Bildung, Wissenschaft, Forschung und Technologie (BMBF, No. 0313965A). We like to thank IBA Göttingen for kindly providing the anti-CD4 (13B.8) Fab sequence.

\section{Competing interests}

The authors declare no competing interests.

\section{References}

1. Chadd, H.E. and S.M. Chamow. 2001. Therapeutic antibody expression technology. Curr. Opin. Biotechnol. 12:188-194.
2. Krauss, J. 2003. Recombinant antibodies for the diagnosis and treatment of cancer. Mol. Biotechnol. 25:1-17.

3. Holliger, P. and P.J. Hudson. 2005. Engineered antibody fragments and the rise of single domains. Nat. Biotechnol. 23:1126-1136.

4. Carter, P.J. 2006. Potent antibody therapeutics by design. Nat. Rev. Immunol. 6:343-357.

5. Kipriyanov, S.M. and F. Le Gall. 2004. Generation and production of engineered antibodies. Mol. Biotechnol. 26:39-60.

6. Humphreys, D.P. 2003. Production of antibodies and antibody fragments in Escherichia coli and a comparison of their functions, uses and modification. Curr. Opin. Drug Discov. Dev. 6:188-196.

7. Yamaji, H., T. Manabea, K. Watakabe, M. Muraokab, I. Fujii, and H. Fukudab. 2008. Production of functional antibody Fab fragment by recombinant insect cells. Biochem. Eng. J. 41:203-209.

8. Hoogenboom, H.R. 2005. Selecting and screening recombinant antibody libraries. Nat. Biotechnol. 23:1105-1116.

9. Spirin, A.S., V.I. Baranov, L.A. Ryabova, S.Y. Ovodov, and Y.B. Alakhov. 1988. A continuous cell-free translation system capable of producing polypeptides in high yield. Science 242:11621164.

10. Strey, J., H. Merk, and W. Stiege. 2004. In vitro synthesis of expression products, particularly proteins, in a cell-free system, comprises using a repetitive batch method, with periodic removal of metabolites and supplementation. patent DE102004032460.

11. Kim, T.W., I.S. Oh, J.W. Keum, Y.C. Kwon, J.Y. Byun, K.H. Lee, C.Y. Choi, and D.M. Kim. 2007. Prolonged cell-free protein synthesis using dual energy sources: combined use of creatine phosphate and glucose for the efficient supply of ATP and retarded accumulation of phosphate. Biotechnol. Bioeng. 97:1510-1515.

12. Madin, K., T. Sawasaki, T. Ogasawara, and Y. Endo. 2000. A highly efficient and robust cell-free protein synthesis system prepared from wheat embryos: plants apparently contain a suicide system directed at ribosomes. Proc. Natl. Acad. Sci. USA 97:559-564.

13. Tsuboi, T., S. Takeo, H. Iriko, L. Jin, M. Tsuchimochi, S. Matsuda, E. Han, H. Otsuk, et al. 2008. Wheat germ cell-free system-based production of malaria proteins for discovery of novel vaccine candidates. Infect. Immun. 76:1702-1708.

14. Tarui, H., M. Murata, I. Tan, S. Imanishi, S. Nishikawa, and T. Hara. 2001. Establishment and characterization of cell-free translation/ glycosylation in insect cell (Spodoptera frugiperda 21) extract prepared with high pressure treatment. Appl. Microbiol. Biotechnol. 55:446-453.

\section{Cell-able Micropatterned, Polymer Coated Culture Plates}
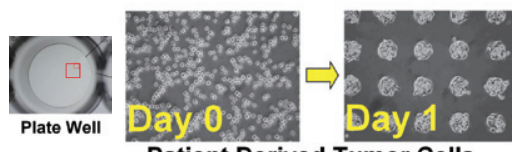

Promotes Spheroid Formation For 3-D Cell Culture

- Primary Tumor Cells, Tumor Lines

- Hepatocytes, fresh or frozen worldwide

USA, Canada www.cosmobio.com

www.cosmobiousa.com
15. Kubick, S., J. Schacherl, H. Fleischer-Notter, E. Royall, L.O. Roberts, and W. Stiege. 2003. In vitro translation in an insect-based cell-free system. p. 209-217. In J.R. Swartz, (Ed.), Cell-Free Protein Expression. Springer-Verlag, Berlin.

16. Mikami, S., M. Masutani, N. Sonenberg, S. Yokoyama, and H. Imataka. 2006. An efficient mammalian cell-free translation system supplemented with translation factors. Protein Expr. Purif. 46:348-357.

17. Ryabova, L.A., D. Desplancq, A.S. Spirin, and A. Pluckthun. 1997. Functional antibody production using cell-free translation: effects of protein disulfide isomerase and chaperones. Nat. Biotechnol. 15:79-84.

18. Merk, H., W. Stiege, K. Tsumoto, I. Kumagai, and V.A. Erdmann. 1999. Cell-free expression of two single-chain monoclonal antibodies against lysozyme: effect of domain arrangement on the expression. J. Biochem. 125:328-333.

19. Tsumoto, K., Y. Nakaoki, Y. Ueda, K. Ogasahara, K. Yutani, K. Watanabe, and I. Kumagai. 1994. Effect of the order of antibody variable regions on the expression of the single-chain HyHEL10 Fv fragment in E. coli and the thermodynamic analysis of its antigen-binding properties. Biochem. Biophys. Res. Commun. 201:546-551.

20. Jiang, X., Y. Ookubo, I. Fujii, H. Nakano, and T. Yamane. 2002. Expression of Fab fragment of catalytic antibody 6D9 in an Escherichia coli in vitro coupled transcription/translation system. FEBS Lett. 514:290-294.

21. Oh, I.S., J.C. Lee, M.S. Lee, J.H. Chung, and D.M. Kim. 2010. Cell-free production of functional antibody fragments. Bioprocess Biosyst. Eng. 33:127-132.

22. Kim, D.M. and J.R. Swartz. 2004. Efficient production of a bioactive, multiple disulfidebonded protein using modified extracts of Escherichia coli. Biotechnol. Bioeng. 85:122-129.

23. Oh, I.S., D.M. Kim, T.W. Kim, C.G. Park, and C.Y. Chloi. 2006. Providing an oxidizing environment for the cell-free expression of disulfide-containing proteins by exhausting the reducing activity of Escherichia coli S30 extract. Biotechnol. Prog. 22:1225-1228.

24. Ezure, T., T. Suzuki, M. Shikata, M. Ito, E. Ando, O. Nishimura, and S. Tsunasawa. 2007. Expression of proteins containing disulfide bonds in an insect cell-free system and confirmation of their arrangements by MALDI-TOF MS. Proteomics 7:4424-4434.

25. Merk, H., D. Meschkat, and W. Stiege. 2003. Expression-PCR: fromgene pools to purified proteins within 1 day.p. 15-23. In Swartz,J.R.,(Ed.), Cell-Free Protein Expression. Springer-Verlag, Berlin.

26. Silverton, E.W., E.A. Padlan, D.R. Davies, S. Smith-Gill, and M. Potter. 1984. Crystalline monoclonal antibody Fabs complexed to hen egg white lysozyme. J. Mol. Biol. 180:761-765.

27. von Groll, U., S. Kubick, H. Merk, W. Stiege, and F. Schäfer. 2007. Advances in insect-based cell-free protein expression. p. 37-48. In W.A. Kudlicki, F. Katzen, and R.P. Bennett (Eds), Cell-free expression. Austin: Landes Bioscience.

Received 3 May 2012; accepted 3 August 2012.

Address correspondence to Helmut Merk, RiNA Netzwerk RNA-Technologien GmbH,Volmerstraße 9, D-12489, Berlin, Germany. Email: Merk@RiNAGmbH.eu

To purchase reprints of this article, contact: biotechniques@fosterprinting.com 